\title{
Double staining immunofluorescence procedure for enumeration of cells containing cytoplasmic immunoglobulin in human bone marrow: 40 selected
}

\author{
cases
}

\author{
MJ A YLIFFE
}

From the Immunology laboratory, St Helier Hospital, Carshalton, Surrey

SUMMARY A double staining immunofluorescence method was developed that allowed the reliable differential counting of cells containing cytoplasmic immunoglobulin in human bone marrow. These cells were stained with a polyspecific antihuman immunoglobulin serum conjugated with rhodamine. In separate preparations subpopulations of cells containing each heavy chain class and light chain type were identified by prior staining with isotype specific fluorescein conjugated antihuman immunoglobulin sera. A standardised counting procedure was adopted to indicate the degree of plasma cell infiltration and to establish the percentage of cells containing each type of immunoglobulin.

A series of 122 patients requiring haematological investigation of the bone marrow for various disorders but without evidence of paraproteinaemia was tested to provide results for a nonmyelomatous reference group. These results were compared with those from 40 patients with paraproteins of various classes in their serum. The procedure described here clearly differentiated the two groups. When combined with serum paraprotein assays and conventional haematological cytology of bone marrow these counts provided useful additional information in cases of diagnostic difficulty and in the assessment of individual patients before, during, and after treatment.

The laboratory diagnosis of myelomatosis relies on the finding of paraprotein in serum or of free immunoglobulin (Ig) light chains in urine, together with the presence of atypical or particularly numerous plasma cells in the bone marrow. Paraproteinaemia can occur in the absence of overt myeloma and without obvious cytological abnormalities in conventional haematological preparations of bone marrow.

Serial measurements of the concentration of paraproteins in the serum and conventional cytological examination of bone marrow are also used to monitor the progress of the disease, but, although they are generally useful, clinical experience shows

Accepted for publication 16 May 1985 them to be misleading in a considerable number of cases.

Immunofluorescence has been used to show the presence of cytoplasmic immunoglobulin (Ig) in plasma cells, ${ }^{1}$ and a counting procedure for the assessment by immunofluorescence of plasma cells in marrow, using fluorescein isothiocyanate anti-Ig conjugates, is used in some centres. ${ }^{2}$ This paper describes a double staining procedure that improved the reliability of such counts. Subpopulations of plasma cells were enumerated in separate preparations according to the class of Ig by staining with monospecific fluorescein isothiocyanate conjugated antisera to Ig heavy chains and light chains and then all plasma cells in every preparation were defined by the presence of cytoplasmic Ig using sheep antihuman Ig serum conjugated with tetramethyl rhodamine isothiocyanate (TRITC) as a counterstain. 


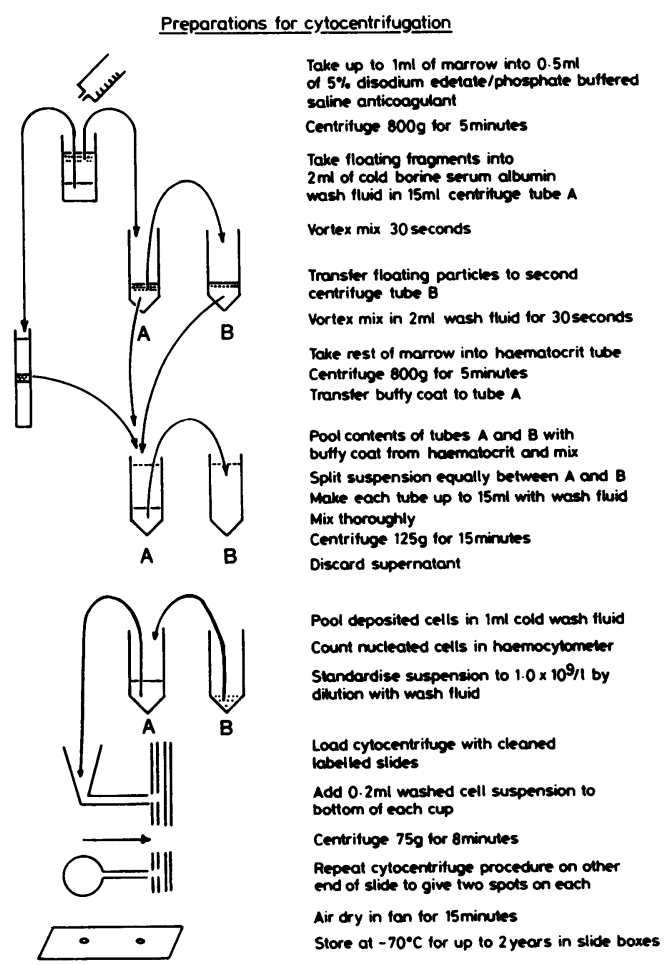

Fig. 1 Preparations for cytocentrifugation

\section{Material and methods}

\section{SAMPLES FROM PATIENTS}

Bone marrow aspirates were collected from 122 inpatients requiring haematological investigation for various reasons; none of them had a serum paraprotein concentration detectable by electrophoresis on cellulose acetate. In addition, samples of marrow were taken from 40 patients with confirmed paraprotein. Fragments of marrow were taken into $0.5 \mathrm{ml}$ of $5 \%$ disodium edetate in phosphate buffered saline ( $\mathrm{pH} \mathrm{7 \cdot 2).} \mathrm{Conventional} \mathrm{marrow} \mathrm{smear}$ preparations were made at the same time.

\section{WASHING FLUID}

Washing fluid was prepared according to the following procedure: phosphate buffered saline $(\mathrm{pH} \mathrm{7.2)}$ $682 \mathrm{ml} ; 5 \%$ disodium edetate in phosphate buffered saline $91 \mathrm{ml}$; and $22 \%$ sterile bovine serum albumin (Biotest, Frankfurt) $227 \mathrm{ml}$. The phosphate buffered saline and disodium edetate were mixed and sterilised by autoclaving at $121^{\circ} \mathrm{C}$ for 20 minutes. After cooling the bovine serum albumin was added and the mixture adjusted to $\mathrm{pH} 6.8$ with sodium bicarbonate powder.

\section{FIXATION}

Slides were fixed in $5 \%$ glacial acetic acid in ethanol for 15 minutes at $-20^{\circ} \mathrm{C}$.

\section{SLIDE PREPARATIONS (Fig. 1)}

Nucleated cells were freed from fragments by vortex mixing, thoroughly washed free of plasma and red blood cells, and deposited on slides using a cytocentrifuge (Shandon, Runcorn).

Samples were centrifuged at $800 \mathrm{~g}$ for five minutes. Floating fragments were taken into $2 \mathrm{ml}$ washing fluid in the $15 \mathrm{ml}$ conical centrifuge tube and mixed vigorously in a vortex for 30 seconds. After standing for two minutes any remaining floating fragments were transferred to a second centrifuge tube containing $2 \mathrm{ml}$ washing fluid and vortex mixed for a further 30 seconds to free cells from fragments. The rest of the marrow was taken into a Wintrobe's/haematocrit tube and spun at $800 \mathrm{~g}$ for five minutes, and the buffy coat was pooled with the suspensions of cells freed from the fragments. These cells were suspended in $30 \mathrm{ml}$ washing fluid in two tubes and gently centrifuged at $125 \mathrm{~g}$ for 15 minutes. The supernatant was discarded and the deposit resuspended in washing fluid at a concentration of $1 \times 10^{9}$ nucleated cells. The cytocentrifuge was loaded with cleaned, labelled slides, and $200 \mu \mathrm{l} / \mathrm{l}$ washed marrow suspension was added to each cup $\left(2 \times 10^{5}\right.$ nucleated cells $)$ and centrifuged at $75 \mathrm{~g}$ for eight minutes.

The cytocentrifuge procedure was repeated on the other end of the slide to give two spot preparations on each slide. Preparations were dried in air in front of a fan for 15 minutes, and each spot was scored around the edge with a diamond pencil to aid later visualisation. Slides were stored in plastic slide boxes with silica gel inside sealed plastic bags at $-70^{\circ} \mathrm{C}$ for up to two years: they were fixed immediately before staining.

\section{FLUORESCENCE CONJUGATES}

Monospecific fluorescein isothiocyanate conjugated antisera to human IgG, IgM, IgA, IgD, $\kappa$ light chains, and $\lambda$ light chains were obtained from commercial sources (Wellcome Diagnostics, Beckenham, and Dakopatts, Denmark). Polyspecific TRITC conjugated antihuman Ig serum kindly donated by Dr S Chantler (Wellcome Reagents) was used as the counterstain, and a TRITC conjugated antibovine serum albumin reagent (Becton Dickinson) provided the control for non-Ig specific rhodamine staining.

\section{CONJUGATE TITRATION}

Fluorescein isothiocyanate conjugates were titrated using preparations of marrow containing normal 
Procedure for bone marrow staining.

Double staining immunof worescence

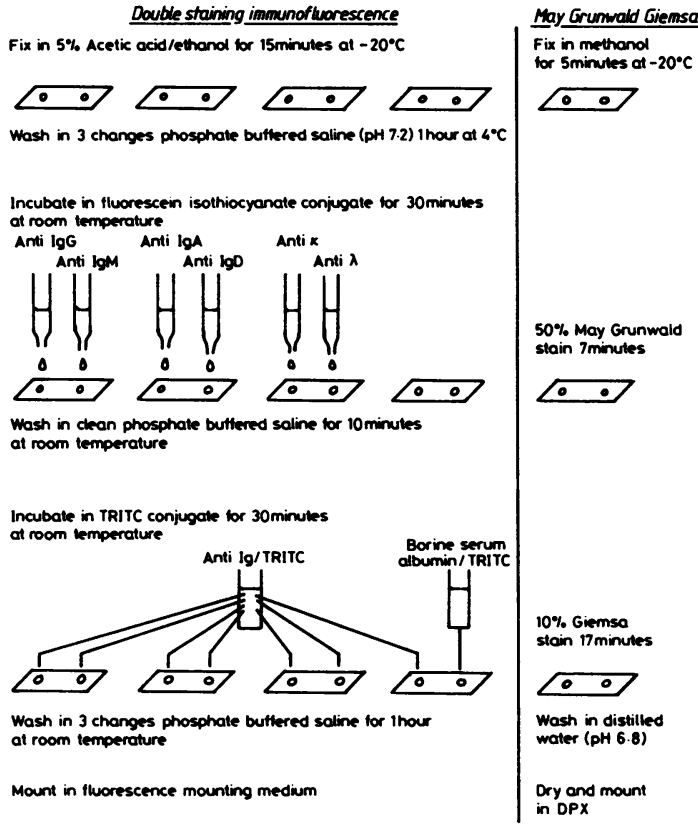

Fig. 2 Procedure for bone marrow staining.

plasma cells to determine their optimal working dilutions. These conjugates were then tested on preparations of myelomatous marrows of known isotype to check their specificity. The dilution for anti-Ig TRITC was determined by titration on preparations optimally stained with each of the fluorescein isothiocyanate conjugated antisera. The antibovine serum albumin TRITC dilution was obtained by comparison with the anti-Ig TRITC reagent, at its optimal dilution for use, by absorbtiometry at $540 \mathrm{~nm}$.

\section{STAINING METHOD (Fig 2)}

Four slides (eight preparations) were transferred from storage boxes to fixative at $-20^{\circ} \mathrm{C}$, taking care to avoid condensation on the unfixed preparations. After fixation for 15 minutes slides were washed in three changes of phosphate buffered saline for one hour, at $4^{\circ} \mathrm{C}$, using a magnetic stirrer. Slides were dried around the preparations, and each preparation was marked with a diamond pencil according to the conjugate it was to receive. Optimally diluted fluorescein isothiocyanate conjugates were centrifuged to clear any deposits and applied to the six appropriate preparations of marrow for $\mathbf{3 0}$ minutes at room temperature in a humid box. (Two preparations were left in phosphate buffered saline during this period as they did not require fluorescein isothiocyanate staining). Fluorescein isothiocyanate conjugates were rinsed off in phosphate buffered saline, and the slides were washed for 10 minutes in phosphate buffered saline with constant mixing to remove unbound antibody. The slides were again dried around the preparations, and the diluted antiIg TRITC reagent was applied to each preparaton. At this point the last two preparations were removed from the phosphate buffered saline wash; the first was stained with anti-Ig TRITC alone and the other with anti bovine serum albumin TRITC as a control. Slides were incubated in rhodamine conjugates for 30 minutes, rinsed in phosphate buffered saline, and finally washed in three changes of phosphate buffered saline for one hour with mixing. The slides were mounted in glycerol/phosphate buffered saline mountant (Citifluor, London).

A separate slide was stained by May Grunwald Giemsa technique for conventional haematological cytology.

\section{MICROSCOPY}

Slides were examined using a Zeiss photomicroscope III fitted with a HBO $50 \mathrm{~W}$ high pressure mercury lamp and an epifluorescence condenser that facilitated selection of both fluorescein isothiocyanate and TRITC filter sets. The filter sets fitted for fluorescein isothiocyanate fluorescence comprised a blue interference exciter filter 455-490, and FT 510 beam splitter, and a 520-560 band pass barrier filter. For TRITC fluorescence a BP 546/7 exciter filter, an FT 580 beam splitter, and an LP 590 red barrier filter were used.A planapochromatic $\times 40$ oil immersion objective was used throughout.

\section{COUNTING PROCEDURE}

The bovine serum albumin TRITC preparation was examined with the TRITC filter set first for evidence of non-Ig specific rhodamine staining. This was usually absent, but in the few cases that gave discernible rhodamine staining this background was taken into account in the assessment of cells stained with antiIg TRITC. The total plasma cell number was determined by counting 500 consecutive nucleated cells in the preparation stained with anti-Ig TRITC alone and recording the number with cytoplasm brightly stained with rhodamine.

The differential count of plasma cells was made by taking each stained preparation in turn and counting 100 consecutive cells containing Ig (red staining) and recording how many were also stained green when examined with the fluorescein isothiocyanate filter set. Thus every cell counted was examined using both filter sets. Morphological observations about stained cells were recorded.

Finally, a parallel preparation stained by the May 
Table 1 Incidence of cells containing cytoplasmic Ig in bone marrow of 122 patients requiring haematological investigation but without paraprotein in their serum

\begin{tabular}{lcc}
\hline & Mean (SEM) (\%) & $\begin{array}{c}\text { Reference range for patients without } \\
\text { paraprotein (mean } \pm 2 S D)(\%)\end{array}$ \\
\hline Cells containing Ig expressed as \% of nucleated cells & $6(0 \cdot 6)$ & $1-11$ \\
Differential counts & & \\
IgG & $53(2 \cdot 0)$ & $31-75$ \\
IgM & $7(1 \cdot 3)$ & $<1-21$ \\
IgA & $37(2 \cdot 3)$ & $12-62$ \\
IgD & $2(0 \cdot 5)$ & $<1-7$ \\
$\kappa$ & $63(1 \cdot 3)$ & $49-77$ \\
$\lambda$ & $37(1 \cdot 1)$ & $25-49$ \\
$\kappa: \lambda$ ratio & $1.73(0 \cdot 08)$ & $0.85-2.61$ \\
\hline
\end{tabular}

Grunwald Giemsa method was examined to correlate cytological features with the immunofluorescence findings.

\section{Results}

Non-Ig specific staining in the bovine serum albumin

TRITC control slide was usually insignificant. Eosinophils and their precursors stained avidly with rhodamine but were easily distinguished from plasma cells by their coarse granularity. Macrophages sometimes stained weakly with both rhodamine and fluorescein conjugates but in a non-Ig specific manner. In the few samples in which

Table 2 Incidence of cells containing cytoplasmic immunoglobulin in bone marrow of 40 patients with paraproteinaemia. together with incidence of distribution according to heavy chain class and light chain type

\begin{tabular}{|c|c|c|c|c|c|c|c|c|c|}
\hline \multirow[t]{2}{*}{ Case No } & \multirow{2}{*}{$\begin{array}{l}\text { Serum } \\
\text { paraprotein }\end{array}$} & \multirow{2}{*}{$\begin{array}{l}\text { Plasma cell } \\
(\%)\end{array}$} & \multicolumn{6}{|c|}{$\%$ of cells of each type } & \multirow{2}{*}{$\frac{\text { Ratio }}{\kappa: \lambda}$} \\
\hline & & & $I g G$ & $\operatorname{Ig} M$ & $\operatorname{Ig} A$ & $I g D$ & $\kappa$ & $\lambda$ & \\
\hline 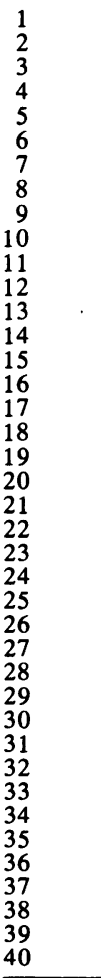 & 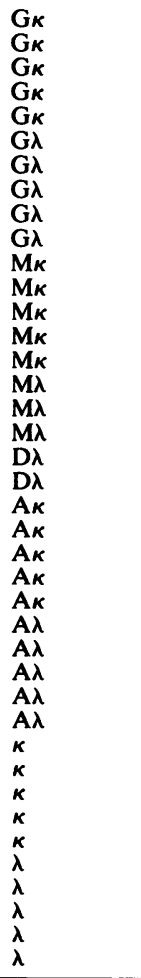 & $\begin{array}{r}34 \\
32 \\
3 \\
45 \\
24 \\
17 \\
16 \\
12 \\
6 \\
17 \\
9 \\
4 \\
14 \\
18 \\
20 \\
11 \\
9 \\
1 \\
57 \\
14 \\
3 \\
2 \\
6 \\
9 \\
17 \\
31 \\
11 \\
81 \\
26 \\
76 \\
54 \\
64 \\
0\end{array}$ & $\begin{array}{r}100 \\
98 \\
97 \\
99 \\
97 \\
98 \\
93 \\
99 \\
78 \\
98 \\
12 \\
3 \\
2 \\
1 \\
2 \\
12 \\
35 \\
44 \\
<1 \\
2 \\
6 \\
6 \\
18 \\
28 \\
11 \\
<1 \\
10 \\
<1 \\
<1 \\
<1 \\
2 \\
6 \\
<1 \\
<1 \\
27 \\
<1 \\
3 \\
1 \\
1 \\
7\end{array}$ & $\begin{array}{r}<1 \\
1 \\
2 \\
<1 \\
1 \\
<1 \\
3 \\
<1 \\
6 \\
<1 \\
87 \\
98 \\
95 \\
97 \\
97 \\
63 \\
38 \\
26 \\
<1 \\
1 \\
2 \\
<1 \\
2 \\
4 \\
3 \\
1 \\
3 \\
<1 \\
<1 \\
<1 \\
<1 \\
<1 \\
<1 \\
<1 \\
11 \\
<1 \\
<1 \\
<1 \\
<1\end{array}$ & $\begin{array}{r}1 \\
1 \\
3 \\
<1 \\
1 \\
2 \\
8 \\
<1 \\
16 \\
<1 \\
1 \\
<1 \\
2 \\
1 \\
3 \\
15 \\
17 \\
28 \\
<1 \\
<1 \\
94 \\
95 \\
86 \\
65 \\
92 \\
99 \\
84 \\
100 \\
100 \\
100 \\
2 \\
33 \\
<1 \\
<1 \\
19 \\
<1 \\
2 \\
<1 \\
<1 \\
7\end{array}$ & $\begin{array}{r}<1 \\
<1 \\
<1 \\
1 \\
<1 \\
<1 \\
<1 \\
<1 \\
<1 \\
<1 \\
<1 \\
3 \\
<1 \\
<1 \\
1 \\
<1 \\
<1 \\
18 \\
2 \\
<1 \\
100 \\
100 \\
96 \\
1 \\
<1 \\
2 \\
2 \\
<1 \\
<1 \\
<1 \\
<1 \\
<1 \\
<1 \\
<1 \\
<1 \\
<1 \\
<1 \\
<1 \\
<1 \\
<1 \\
<1 \\
<1 \\
<1 \\
<1 \\
<1\end{array}$ & $\begin{array}{r}99 \\
97 \\
95 \\
99 \\
99 \\
5 \\
4 \\
<1 \\
34 \\
6 \\
89 \\
96 \\
97 \\
100 \\
96 \\
36 \\
43 \\
48 \\
<1 \\
2 \\
87 \\
96 \\
78 \\
71 \\
93 \\
<1 \\
17 \\
<1 \\
<1 \\
<1 \\
100 \\
95 \\
96 \\
99 \\
79 \\
<1 \\
4 \\
3 \\
1 \\
8\end{array}$ & $\begin{array}{r}<1 \\
1 \\
6 \\
1 \\
1 \\
92 \\
91 \\
98 \\
61 \\
95 \\
6 \\
1 \\
2 \\
2 \\
5 \\
66 \\
58 \\
57 \\
100 \\
90 \\
9 \\
2 \\
18 \\
23 \\
3 \\
100 \\
86 \\
100 \\
100 \\
100 \\
<1 \\
<5 \\
<1 \\
<1 \\
15 \\
100 \\
96 \\
98 \\
99 \\
92\end{array}$ & $\begin{array}{r}>99 \\
97 \\
16 \\
99 \\
99 \\
0.05 \\
0.04 \\
<0.01 \\
<0.56 \\
<0.06 \\
15 \\
96 \\
48 \\
50 \\
19 \\
0.55 \\
0.74 \\
0.94 \\
<0.01 \\
0.02 \\
10 \\
48 \\
4 \\
3 \\
31 \\
<0.01 \\
<0.2 \\
<0.01 \\
<0.01 \\
<0.01 \\
>100 \\
19 \\
>996 \\
>99 \\
9.4 \\
<0.01 \\
0.04 \\
0.03 \\
0.01 \\
0.08\end{array}$ \\
\hline
\end{tabular}


background staining was appreciable it was never as intense as the staining of cells containing $\mathrm{Ig}$ in the anti-Ig stained preparations.

Most cells that stained were of typical plasma cell morphology; some samples, however, contained cells of more lymphocytoid appearance which also contained cytoplasmic immunoglobulin. Occasionally, lymphocytes with a surface membrane distribution of Ig were seen; these were excluded from the counts, although their presence was recorded.

The mean value for the incidence of cells containing cytoplasmic immunoglobulin in patients without serum paraprotein by this method was 6\% (SEM $0 \cdot 6)$. Table 1 presents the results for the differential counts, together with the ratio of cells containing $\kappa$ and $\lambda$ light chains. A reference range derived from these results facilitated comparison when the results in each patient were assessed (Table 1).

Table 2 shows the results obtained from testing the bone marrow of patients with readily detectable paraprotein in their serum together with the immunochemical class of their paraprotein.

\section{Discussion}

Immunochemical assays of serum and urine, together with haematological assessment of bone marrow, are widely used in the investigation of myeloma and monoclonal gammopathies of undetermined significance. The presence of cytoplasmic Ig in bone marrow cells shown by immunofluorescence has been used to elucidate cases in which diagnosis has been difficult. ${ }^{3}$

In the original method separate preparations were stained with monospecific fluorescein conjugated antisera to each Ig heavy chain and light chain type. ${ }^{2}$ The cells staining in each heavy chain stained preparation were counted and expressed as a percentage of the total counted in all the heavy chain preparations. Similar procedures were carried out for the light chain counts. Thus the sums of the heavy chain counts and of the light chain counts were always $100 \%$. Alternatively, the stained cells could be counted in a defined number of optical microscope fields or the counts for each preparation could be expressed as a total of the nucleated cells seen.

These counts showed monoclonal proliferations in most cases in which they existed, but inaccuracies resulting from the differences in the distribution of cells between one preparation and another limited their value in showing fine variations of pathological importance and in the assessment of progression of disease or response to treatment.

The procedure described here overcame these objections in that the fluorescein isothiocyanate stained cells in each preparation were counted as a proportion of the cells containing $\mathrm{Ig}$, as defined by staining with anti-Ig TRITC in that same preparation. The sum of the heavy chain percentages and the sum of the light chain types were usually around $100 \%$; if they were outside the range $90-110 \%$ then the tests were repeated or an explanation (such as free light chain disease) was sought. The double staining procedure thus increased accuracy and provided an element of internal control. Double staining of single cells with easily differentiated colours for different determinants represented an advantage of immunofluorescence over conventional enzyme immunohistochemical methods. ${ }^{4}$

Attention to technical details was essential in handling the bone marrow cells, particularly in washing them free of plasma and standardising their concentration before making the slide preparations. The method was economical in reagents, and the commercially available conjugated antisera were reliable. Although it was acceptable to use sera from different species, there was some degree of cross reaction which enhanced the second antibody staining when a first antibody (rabbit) and second antibody (sheep) were used. It was therefore preferable to use antisera raised in the same species. The procedure for staining could be shortened with only a slight loss in clarity by applying both the fluorescein isothiocyanate and TRITC conjugated antisera as a mixture at the same time; equal parts of both reagents were mixed at twice the optimal concentration used in the recommended procedure.

Ethical considerations precluded the establishment of a range of reference values derived from normal healthy subjects for comparison with patients in these tests, and it may be that such values would not be particularly appropriate to clinical practice. The figures for subjects without a paraprotein presented here are the results from a cross section of patients presenting for bone marrow cytology as part of their haematological investigation in a district general hospital.

The figures for total plasma cells were generally slightly higher than those obtained at cytological examination because the criteria were differentthat is, in this investigation plasma cells were defined as cells with a cytoplasm that stained brightly for Ig. The differential figures show the cells that produce IgG to have the widest range.

IgM cells are relatively uncommon in human bone marrow, suggesting that the marrow is not a principal site for the production of IgM. Experiments on mice have shown that production of marrow antibody is much less evident in primary (usually $\operatorname{IgM}$ ) immune responses than that in secondary responses. $^{5}$ The low incidence of cells containing IgM in human bone marrow could also account for 
the fact that bone marrow involvement is relatively minor in Waldenstrom's macroglobulinaemia (an IgM malignancy) and that the incidence of lymphadenopathy and organomegaly in such cases is increased compared with that in patients with IgG and IgA myeloma. ${ }^{\circ}$

Cells containing IgD were the least common in the marrow, but the mean figure of $2 \%$ was in general agreement with the reported incidence of $\operatorname{IgD}$ myeloma.? The range of $1-7 \%$ for the frequency of plasma cells containing $\operatorname{IgD}$ was a little surprising, considering the low serum concentration of $\operatorname{IgD}$ and the fact that no clearly accepted biological role has yet been established for humoral IgD.

The figures for $\kappa$ and $\lambda$ light chains were those expected from the serum concentrations. The range for the ratio of $\kappa$ to $\lambda(0.85-2.61)$ was narrower than that quoted for the single fluorescein isothiocyanate staining method $(1 \cdot 0-6 \cdot 0)$, and this may have reflected the improved precision of the double staining and counting procedure reported here. The ratio of $\kappa$ to $\lambda$ counts was the most sensitive indicator of monoclonal proliferation by this method.

Cytoplasmic immunofluorescence counting of bone marrow cells clearly showed the difference between those patients with paraproteinaemia and those without paraprotein in their serum. In some patients (for instance, cases 3 and 9 in the group with IgG paraprotein) the overall number of plasma cells was not increased but differential counting of those plasma cells according to class of Ig clearly indicated monoclonal proliferation. Thus it was advisable to consider all the counted results in the overall interpretation of individual cases. The method clearly differentiated reactive plasmacytoses from proliferations of monoclonal plasma cells, and in this respect the ratio of $\kappa$ to $\lambda$ counts was the most important indicator.

Cells containing IgM formed a relatively small proportion of the plasma cells in human bone marrow, and in macroglobulinaemia the marrow was less involved than in IgG and IgA myelomas. For these reasons a significant increase in cells secreting monoclonal IgM was therefore less obvious than in those secreting IgG and IgA. For instance, a patient (case 19 ) in the group with IgM had only $1 \%$ plasma cells and the $\kappa$ to ratio was within the reference range for cases without paraprotein.
Patients with monoclonal proliferations affecting cells restricted to the production of free light chains clearly had a discrepancy between the sums of their heavy chain and light chain counts.

In the diagnosis of myelomatosis and the assessment of paraproteinaemia adequate, readily available tests are required that will indicate the extent of the tumour burden and the stage and rate of progression of disease and that will measure responses to treatment. The double staining immunofluorescence investigation of bone marrow aspirates described here offered an additional source of information that was useful in the assessment of patients with myeloma and associated disorders. The usefulness of this procedure in cases of diagnostic difficulty and in the management of individual cases will be reported separately.

I thank Professor JG Hall, Royal Marsden Hospital, for advice and helpful discussion in the preparation of this paper.

\section{References}

' Vasquez JJ. Antibody- or gamma globulin-forming cells, as observed by the fluorescent antibody technique. Lab Invest 1961;10:1110-24.

${ }^{2}$ Hijmans W, Schuit HRE, Klein F. An immunofluorescence procedure for the detection of intracellular immunoglobulins. Clin Exp Immunol 1969;4:457-72.

${ }^{3}$ Hijmans W, Schuit HRE, Hulsing-Hesselink E. An immunofluorescence study on intracellular immungloblins in human bone marrow cells. Ann NY Acad Sci 1971;177:290305.

${ }^{4}$ Cordell JL, Falini B, Erber WN, et al. Immunoenzymatic labelling of monoclonal antibodies using immune complexes of alkaline phosphatase and monoclonal anti-alkaline phosphatase (APAAP complexes). J Histochem Cytochem 1984 32:219-29.

s Benner R, Meima F, Van der Meulen GM, Van Muiswinkel WB. Antibody formation in mouse bone marrow. Immunology 1974; 26: 247-55.

- McCallister BD, Bayrd ED, Harrison EG, McGuckin WF. Primary macroglobulinaemia. Review with a report on thirty-one cases and notes on the value on continuous chlorambucil therapy. Am J Med 1967; 43:394-434.

7 Ameis A, Ko HS, Pruzanski W. M components-a review of 1242 cases. Can Med Assoc J 1976;144:889-95.

Requests for reprints to: Mr MJ Ayliffe, Department of Immunology, St Helier Hospital, Wrythe Lane, Carshalton, Surrey SM5 1AA, England. 